\title{
Searching Algorithm of Dormant Node in Wireless Sensor Networks
}

\author{
https://doi.org/10.3991/ijoe.v13i05.7054 \\ Bo Feng \\ Shaanxi University of Science \&Technology, Shaanxi Xi'an, China \\ ckmtvxo3434@126.com \\ Wei Tang \\ Shaanxi University of Science \&Technology, Shaanxi Xi'an, China \\ yicliuy $8558646 @ 126$. com \\ Guofa Guo \\ Shaanxi University of Science \&Technology, Shaanxi Xi'an, China \\ xavrh 752594 @ 126. com
}

\begin{abstract}
In wireless sensor networks, the nodes around the base station have higher energy consumption due to the forwarding task of all the detected data. In order to balance the energy consumption of the nodes around the base station, a reasonable and effective mechanism of node rotation dormancy is put forward. In this way, a large number of redundant nodes in the network are in a dormant state, so as to reduce the load of important nodes around the base station. The problems of the redundant nodes in the sensor network are analyzed, and a new method is proposed to distinguish the redundant nodes based on local Delaunay triangulation and multi node election dormancy mechanism. The experimental results showed that this method could effectively distinguish the redundant nodes in the network; at the same time, through the multi round election mechanism, parts of redundant nodes are made dormant. In summary, they can reduce the network energy consumption on the condition of guaranteeing the original coverage.
\end{abstract}

Keywords - wireless sensor, redundant node dormancy, Delaunay triangulation

\section{Introduction}

Wireless sensor network is the multi hop and self-organizing network system composed through wireless communication of a large number of nodes with a probability distribution model randomly deployed in the monitoring region [1]. Wireless sensor technology has been gradually extended from the earliest military defense applications to agriculture, environmental monitoring, biological medicine, traffic management, remote management of dangerous areas and even home and other fields [2-3]. For high density network application, there are a large number of dormant redundant nodes. We can design the redundant node identification algorithm, to make parts of 
the nodes have alternate dormancy without affecting the network coverage and connectivity requirements, thus reducing the flow for sending data flow to an important node, and saving the energy of essential nodes. For the important nodes, the energy consumption level is higher, can dispenser the relay node around an important node and help the important node to share the task of transmitting large amount of data flow. In this way, it can guarantee the service life of an important node. The method of solving this problem at present mainly focuses on designing an effective MAC protocol, identifying the redundant nodes and making it dormant, so as to ensure prolonging the life cycle of important nodes and design a rational network topology and node rotation dormancy [4].

In this paper, by analyzing the problem of the redundant nodes in the sensor network, we put forward a new distributed redundant node discrimination method based on the local Delaunay triangulation graph and the mechanism of multi node election. The results showed that this method can effectively distinguish the redundant nodes in the network; at the same time, through the multi round election mechanism, we make part of redundant nodes dormant and reduce the network energy consumption on the condition of guaranteeing the original coverage.

\section{State of the art}

For wireless sensor networks, it is two important goals to balance the energy consumption of the sensor nodes and improve the life cycle of the network. The distribution of sensor nodes in a topology affects the energy consumption of each node. In the process of transmitting information, due to the different location of the node, the energy loss is also different. In 2009, Xu Gang et al. proposed application-level node dormancy / synchronization mechanism, and provided a method to calculate the empty proportion based on the expected lifetime and data acquisition requirements of nodes. The results show that the lifetime of the nodes is prolonged. With sleep technology, 150h of no sleep node life can reach more than 1 year. In 2013, Hong Lu et al. proposed a dormancy algorithm for underwater sensor network nodes based on tree topologies. The algorithm can effectively shorten the number of wake-up times, prolong the sleep time, and ensure that the end-to-end propagation delay is not affected by the sleep time. In 2015, Chen Long and other proposed a comprehensive sensor network clustering and node sleep mechanism of the agreement. It divides the life of the sensor network into several time periods. In each time period, it determines the set of similar nodes for each node, and selects the representative node (Rnode) to send the perceived data. In addition, it also hides some redundant nodes. The results show that the protocol can extend the life of the sensor network. In 2016, Feng Lin proposed a border area deployment adjustment of hybrid sensor network node optimization algorithm. By adding a small number of mobile nodes to the divided boundary area, it achieves the energy balance in the process of node scheduling, and realizes the purpose of extending the network lifetime.

In summary, the above researches are aimed at prolonging the network lifetime of sensor networks. In order to balance the energy consumption of the nodes around the 
base station, a reasonable and effective mechanism of node rotation dormancy is put forward. In this way, a large number of redundant nodes in the network are in a dormant state, so as to reduce the load of important nodes around the base station. The problems of the redundant nodes in the sensor network are analyzed, and a new method is proposed to distinguish the redundant nodes based on local Delaunay triangulation and multi node election dormancy mechanism.

\section{Problem of redundant coverage and related algorithms}

Because of the wireless sensor network node hardware platform with limited resources, huge amount of network nodes amount and complex practical application environmental conditions and not allowing to replace the battery of "failure" nodes, it is an effective measurement for saving the nodes with limited battery energy and trying to prolong the whole network by reducing the energy consumption of the nodes. It is implemented by reducing the redundant broadcasting amount and making more nodes in the dormant state in the case of ensuring complete coverage. The reason is that the energy consumption of wireless communication module nodes accounted for more than $90 \%$ of the total energy consumption, the wireless communication module has the maximum energy consumption in the sending state, followed by the receiving state and the idle state, and the energy consumption of dormant state is the lowest. At present, when the communication module of the commonly used Berkeley Motes sensor is in the transmitting state, the power consumption is $60 \mathrm{~mW}$, when in receiving state and idle state, the power consumption is $12 \mathrm{~mW}$, and in the dormant state, the power consumption is only $0.03 \mathrm{~mW}$. Therefore, it will make parts of the nodes in the dormant state and greatly reduce the energy consumption of the nodes and prolong the working time of the nodes under the premise of satisfying the coverage requirements.

The premise of the redundant node dormancy algorithm is the determination of redundant nodes, that is to say, the nodes judge whether the covered area can be completely detected by the neighbor nodes according to the information of neighbor nodes. After the judgment of redundant nodes, because it is a distributed algorithm, each node, only based on the neighbor information, judges whether it belongs to the node. However, since that the correlation of the node coverage is not that each node can be dormant, for example in Figure 1, node 5 and node 6 can determine itself as redundant nodes but not dormant at the same time of node 5 and node 6 , otherwise, between node 1, 2, 3 and 4, it will produce the coverage holes. Therefore, how to design the redundant node algorithm is an important part of the research on the coverage problem of wireless sensor networks.

At present, the main algorithms for judging redundant nodes are Di Tian algorithm [5] and CCP algorithm [6]. The study found that these algorithms have a certain degree of problems. In the following, we will analyze and elaborate in details. 

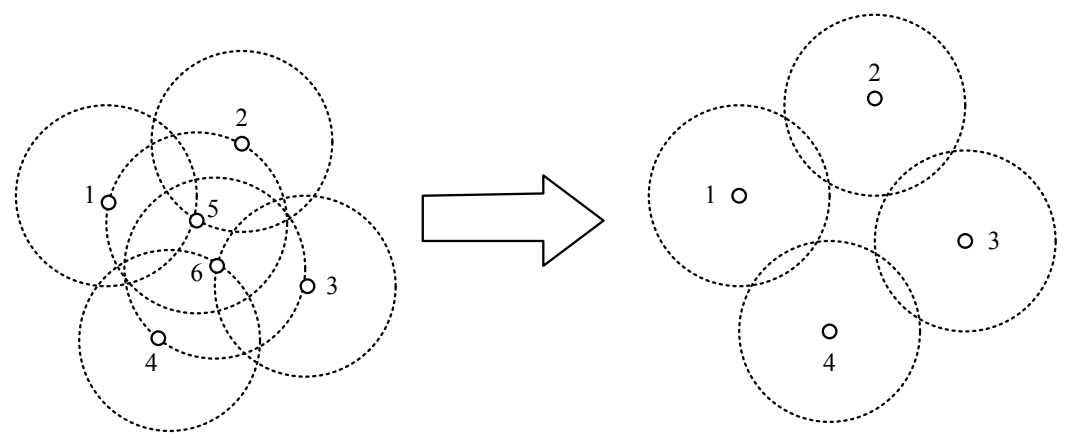

Fig. 1. Coverage holes

The idea of Di Tian algorithm is that if a node is covered by all the neighbor nodes $[0,2 \pi]$, then the node is a redundant node. Specific as follows: for any node $\mathrm{v}$, define its neighbor node set $\mathrm{N}(\mathrm{v})=\left\{\mathrm{u} \mid \mathrm{d}(\mathrm{u}, \mathrm{v}) \leq \mathrm{r}_{\mathrm{s}}\right\}, \mathrm{d}(\mathrm{u}, \mathrm{v})$ indicating the distance from $u$ to $v$. Define the open angle of the neighbor node $u$ to $v$ as $\beta_{u i \rightarrow v}$, as shown in Figure $2 \angle \mathrm{P}_{1} \mathrm{AP}_{2}$.

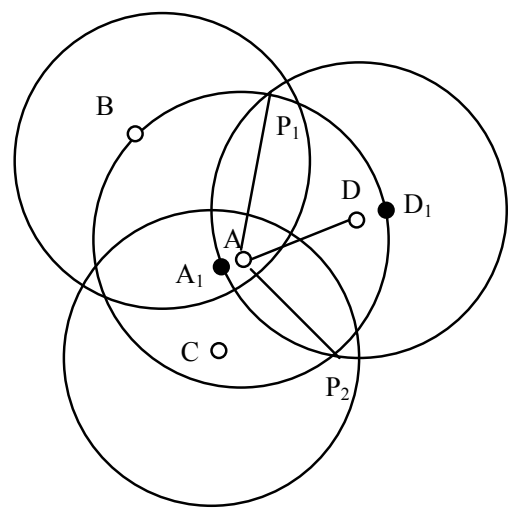

Fig. 2. Di Tian algorithm

If all the neighbor nodes of the node $\mathrm{v}$ to the set of the open angle of $\mathrm{v}$ covers $[0,2 \pi]$, that is $\bigcup_{\mathrm{u}_{\mathrm{i}} \in \mathrm{N}(\mathrm{v})} \beta_{\mathrm{ui} \rightarrow \mathrm{v}}=[0,2 \pi]$, then the node $\mathrm{v}$ is a redundant node.

In Figure 2, Node $\mathrm{A}$ is covered by $\mathrm{B}, \mathrm{C}$, and $\mathrm{D}$ because $\angle_{\mathrm{B} \rightarrow \mathrm{A}} \cup \angle_{\mathrm{C} \rightarrow \mathrm{A}} \cup \angle_{\mathrm{D} \rightarrow \mathrm{A}}=[0,2 \pi]$. In the Di Tian algorithm, because of using the opening angle to judge the coverage, it makes each node, in considering whether or not covered, only considers the nodes with the distance not greater than the radius of the probe, which makes the set of the redundant nodes calculated tends to be small. 
In Figure 3, the area of node $\mathrm{A}$ is completely covered by $\mathrm{B}, \mathrm{C}$, and $\mathrm{D}$. But $\mathrm{D} \notin \mathrm{N}$ ( A ), according to Di Tian algorithm, A cannot be covered by B and C, so we determine that $\mathrm{A}$ is not redundant nodes, which makes the wrong judgment, and makes the finally judged redundant nodes are small in number.

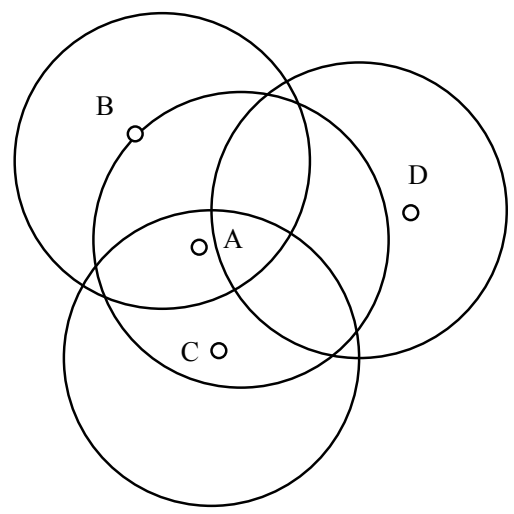

Fig. 3. Wrong judgment in Di Tian algorithm

The detection range of two nodes $u$ and $v$ is overlapped, and the condition needs to meet is that its distance is less than two times of the detection distance, namely $\mathrm{d}(\mathrm{u}, \mathrm{v}) \leq 2 \mathrm{r}_{\mathrm{s}}$. In order to solve the problem of Di Tian algorithm, CCP algorithm defines the neighbor nodes set of any node $v$ as $N(v)=\left\{u \mid d(u, v) \leq r_{s}\right\}$, which enlarges the search range and improves the accuracy of the judgment.

The judgment idea of CCP algorithm is based on a theory in the graph theory. That is to say, in the circle $\mathrm{C}(\mathrm{v})$ of taking node $\mathrm{v}$ as the center, and detection radius $\mathrm{r}_{\mathrm{s}}$ as the radius, the intersection of any two neighbor nodes is always covered by the third neighbor node, then the detection area of node $\mathrm{v}$ is covered by its neighbor nodes, that is, $\forall \mathrm{P} \in[\mathrm{C}(\mathrm{x}) \cap \mathrm{C}(\mathrm{y})], \exists \mathrm{z}$ and $\mathrm{P} \in \mathrm{C}(\mathrm{x}) \mid\{\mathrm{x}, \mathrm{y}, \mathrm{z}\} \subseteq \mathrm{N}(\mathrm{v})$. This algorithm does not have any error, and it will not judge redundant nodes with missing. The only problem is that the computational complexity is too high, analyzing as follows. It is assumed that the node $\mathrm{v}$ has $\mathrm{n}$ neighbor nodes, then the circle intersection of these neighbor nodes is up to $C_{n}^{2}$ intersections, and the amount level is $\mathrm{o}\left(\mathrm{n}^{2}\right)$. The node $v$ first of all calculates the node position of $\mathrm{o}\left(\mathrm{n}^{2}\right)$, then conducts its traversal of $\mathrm{n}-2$ neighbor nodes, to determine whether the cross point is covered by the third party node. Its computation complexity is $\mathrm{o}\left(\mathrm{n}^{3}\right)$, and this calculation complexity in the high density network is very high. For instance, $n=20$, the computational complexity is reached o (8000) level, and the CPU computing capability of sensor nodes is limited, which makes the algorithm not applicable in wireless sensor networks. 
In the aspect of redundant node algorithm, Nicolas D. Georganas proposed a "qualified sleep criterion" for random delay messages to make the redundant nodes dormant, (COBS) algorithm [7-8], as shown in Figure 4:

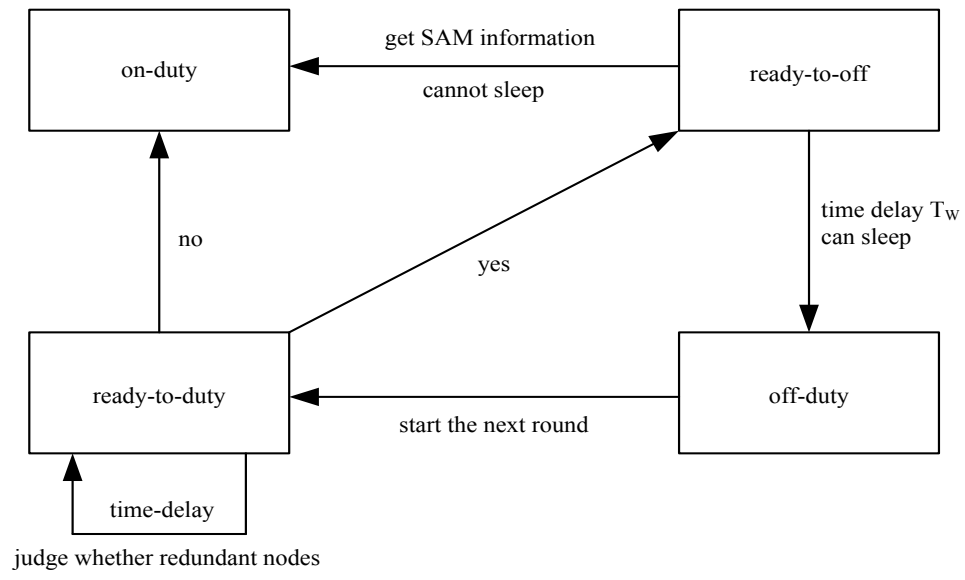

Fig. 4. Sketch map of COBS algorithm

COBS algorithm makes use of a random delay of the multi round selection mechanism, and in each round, all nodes in the network need to determine whether it is a redundant node and whether it can be dormant. As shown in Figure 4, the node has a total of four states, that is, ready-on-duty (preparatory work), on-duty (work), readyto-off and off-duty. At the beginning of each round, all nodes are in the ready-on-duty state. At the beginning of the algorithm, each node generates a random delay $T_{d}$, and in $T_{d}$, finishes to determine whether they are redundant nodes. If not, directly enter the on-duty state for continuing the work; if it is, then enter the ready-to-off (pre dormancy) state and generate a random delay $\mathrm{T}_{\mathrm{w}}$.

In the process of $\mathrm{T}_{1} \mathrm{~T}_{\mathrm{w}}$, if the node receives the message and cannot sleep, then the node directly enters the on-duty (working) state. To the end of $\mathrm{T}_{\mathrm{w}}$, the node determines whether they can sleep based on the coverage situation at that time, that is, whether the detection area of its own is detected by the neighbor nodes. If judging that their dormancy does not generate new coverage holes, then the node enters the off-duty (dormant) state. That is to say, the node gets dormant and does not participate in the next round of selection, and transmits a message notification to the neighbor node in the meanwhile. If determining that the dormancy will create a new vulnerability, then the node enters the on-duty state and continues to work.

The COBS algorithm applies the random delay dormancy of redundant nodes, largely avoiding the redundant nodes and at the same time dormancy related coverage vulnerability, but because the delay generated is uncertain, and the process of infor- 
mation processing of nodes will consume a certain time, the misjudgment situation may still occur, that is to say, it can be qualified dormancy in a particular time node.

\section{$4 \quad$ Algorithm design}

In this paper, the design of the algorithm is divided into two parts, namely: (1) to identify the redundant nodes; (2) in the case of ensuring the original coverage, make dormant of the redundant nodes.

(1) Distinguish the redundant nodes

In the randomly deployed sensor nodes, each node, according to the relative position of the neighbor node information, first of all constructs local Delaunay triangle graph. This paper will adopt the method of establishing the local Delaunay triangle graph in distributed environment. The node, according to the relative position of the nodes in the communication range (i.e., neighbor nodes), constructs the corresponding perpendicular bisector of the line segment. The smallest convex polygon surrounded by the bisector is Voronoi polygon corresponding to that node, the corresponding node is Delaunay neighbor nodes of the node, so it can get local Delaunay triangle graph.

In this algorithm, we use two different concepts: neighbor node and Delaunay neighbor node:

Neighbor nodes: all nodes with distance to the node $i$ less than $R_{C}$ are called neighbor node of the node $i$.

Delaunay neighbor node: in the local Delaunay triangulation graph constructed by the node i, all nodes of the Delaunay triangle edge with the node $i$.

Based on the analysis of local Delaunay triangle graph, this paper proposes a new criterion for distinguishing redundant nodes:

For any node $\mathrm{S}_{\mathrm{i}}$, it there does exist $\max \left\{\mathrm{s}_{\mathrm{i}}\right.$ Delaunay triangle neighbor line length $\} \leq \mathrm{R}_{\mathrm{s}}$, the node $\mathrm{S}_{\mathrm{i}}$ is the redundant node. As shown in Figure 5, the maximum distance of the node to the $\mathrm{S}_{6}$ Delaunay neighbor nodes $\mathrm{S}_{2}, \mathrm{~S}_{4}, \mathrm{~S}_{5}, \mathrm{~S}_{7}$, and $\mathrm{S}_{9}$ is $\overrightarrow{\mathrm{S}_{6} \mathrm{~S}_{5}}$ and $\left\|\overrightarrow{\mathrm{S}_{6} \mathrm{~S}_{5}}\right\|<\mathrm{R}_{\mathrm{S}}$, so $\mathrm{S}_{6}$ is distinguished as the redundant node.

(2) Make redundant nodes dormant in the case of ensuring the coverage unchanged

According to the criteria in (1), the redundant nodes in each node just starts from their own situation, and do not consider other nodes. That is to say, if some redundant nodes are dormant at the same time, it will produce network coverage holes. In this algorithm, the redundant nodes judged by (1) is divided into independent redundant nodes and dependent redundant nodes two categories:

Dependent redundant nodes: if Delaunay neighbor node of a redundant node centralized contains at least one redundant node, the redundant nodes are dependent redundant nodes; the number of Delaunay neighbor nodes is called dependence. 


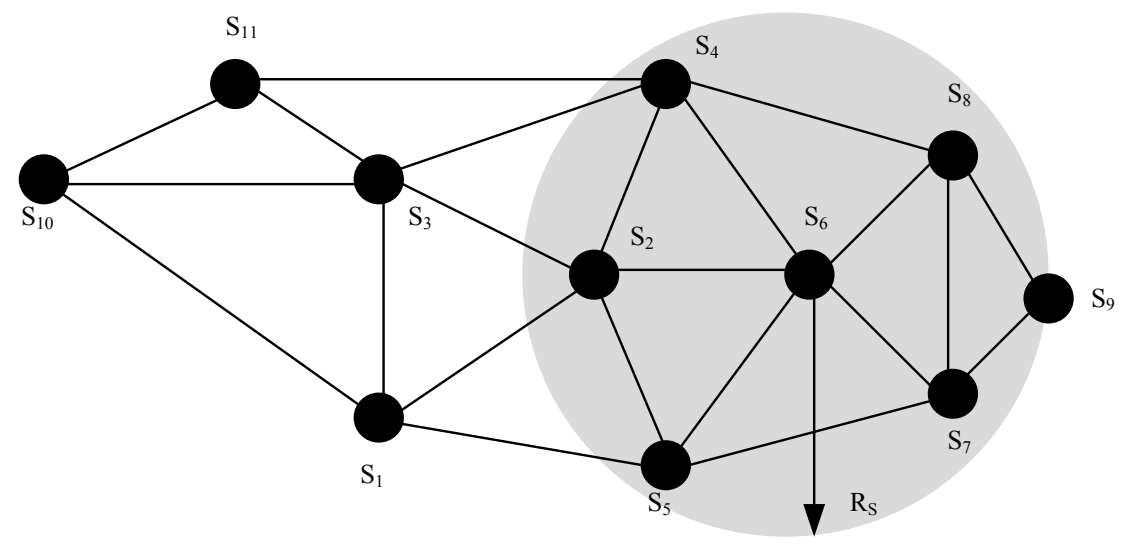

Fig. 5. The judgment of redundant node

Independent redundant node: if all of its Delaunay neighbor nodes are nonredundant nodes, then the redundant nodes are independent redundant nodes.

Independent redundant nodes can go directly to the dormant state, and for the dependent redundant nodes, direct dormancy will lead to the formation of the coverage holes. As a result, we can only choose parts of the nodes to enter the dormant state. In this paper, we propose a new algorithm for the selection of dormancy: first of all, the redundant nodes are grouped according to the Delaunay correlation, and then the multiple voting mechanism is implemented in each group to select the redundant nodes that can get dormant at the same time. In each round, active redundant nodes within a group send the news containing its own ID and dependence to the other node within the group, while receive news released by other nodes, and compare the dependence of the messages received with that of itself. If its dependence is the lowest in the node received, then it will be judged as dormant redundant nodes, while sending a message to the Delaunay neighboring nodes to give a statement of itself dormancy and entering the dormancy state. The node that receives the dormant message remains active state and does not participate in the election any more, that is, not handling the relevant messages. All the active redundant nodes that do not receive the dormancy message enter the next election. The schematic diagram of the process is shown in Figure 6.

$\mathrm{S}_{1} \sim \mathrm{S}_{7}$ in Figure 6 has been determined to be dependent redundant node. From the definition above, the dependency of $\mathrm{S}_{1}, \mathrm{~S}_{4}$, and $\mathrm{S}_{7}$ is 1 , and the dependency of $\mathrm{S}_{2}, \mathrm{~S}_{3}$, and $\mathrm{S}_{6}$ is 2 , and the dependence of $\mathrm{S}_{5}$ is 3 , as shown in Figure 6 (a). In the first round of the election, $\mathrm{S}_{2}, \mathrm{~S}_{3}$, and $\mathrm{S}_{6}$ enter a dormant state, and send message to Delaunay neighbor nodes $\mathrm{S}_{2}, \mathrm{~S}_{3}$, and $\mathrm{S}_{6}, \mathrm{~S}_{2}, \mathrm{~S}_{3}$, and $\mathrm{S}_{6}$ receive the news, that 
is, to maintain the active state, and no longer participate in the elections, and $\mathrm{S}_{5}$ enters the next round of elections, as shown in Figure 6 (b). As there is only $\mathrm{S}_{5}$ left to participate in the election, $S_{5}$ is directly into the dormant state, as shown in Figure 6 (c).

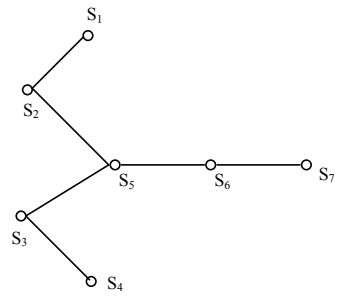

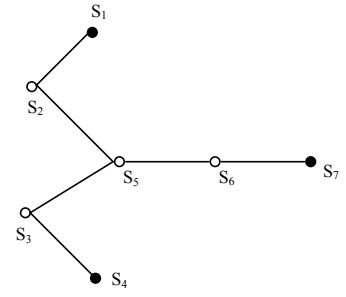

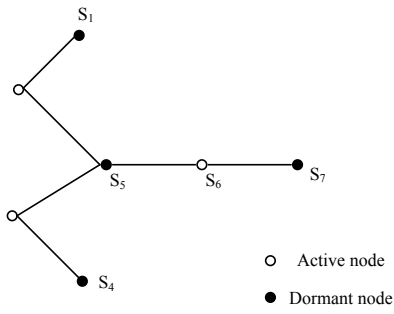

Fig. 6. Schematic diagram of multi round election mechanism

\section{$5 \quad$ Experimental results and analysis}

Figure 7 shows the network topology of this study.

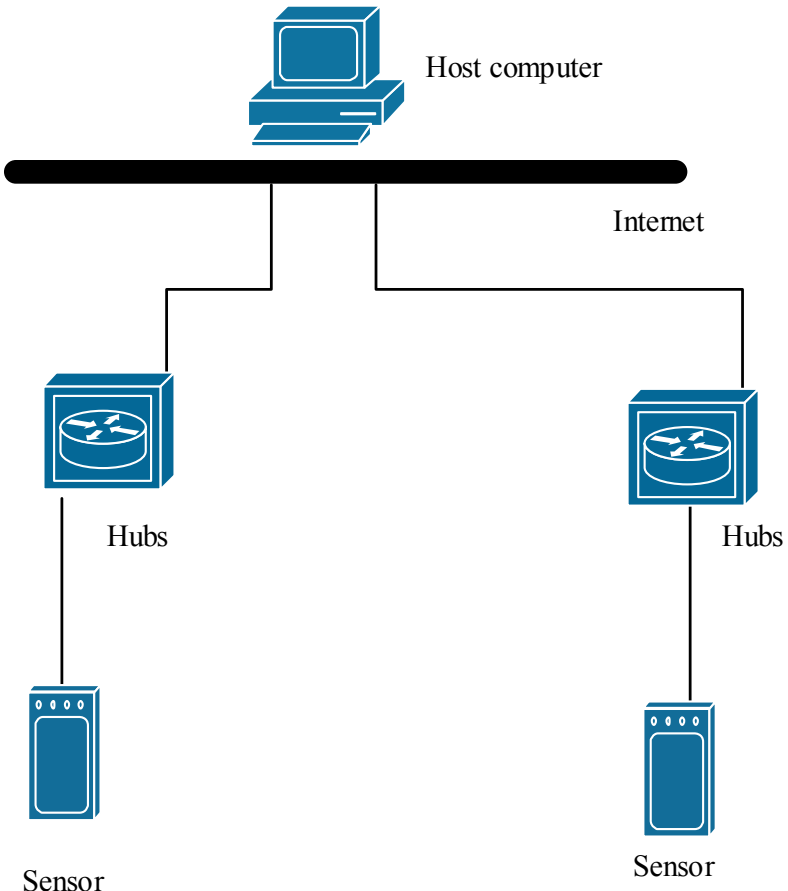

Fig. 7. Network topology diagram 
This paper has several dispenser of different number of nodes in the same coverage area, and from the following two aspects, compares the existing algorithms and the algorithm in this paper:

1. the proportion of redundant nodes to all nodes: calculate the proportion of the number of judged redundant nodes selected by the algorithm of the total number.

2. the number of active nodes: calculate, in the case of different dispenser of the number of nodes within the same region, the number of active nodes that a variety of dormancy algorithms select.

Figure 7 and Figure 8 show, in the case of different dispenser of the number of nodes within the same region, the proportion of redundant nodes accounted for the total number of nodes and the proportion of redundant nodes in the total number of active network nodes.

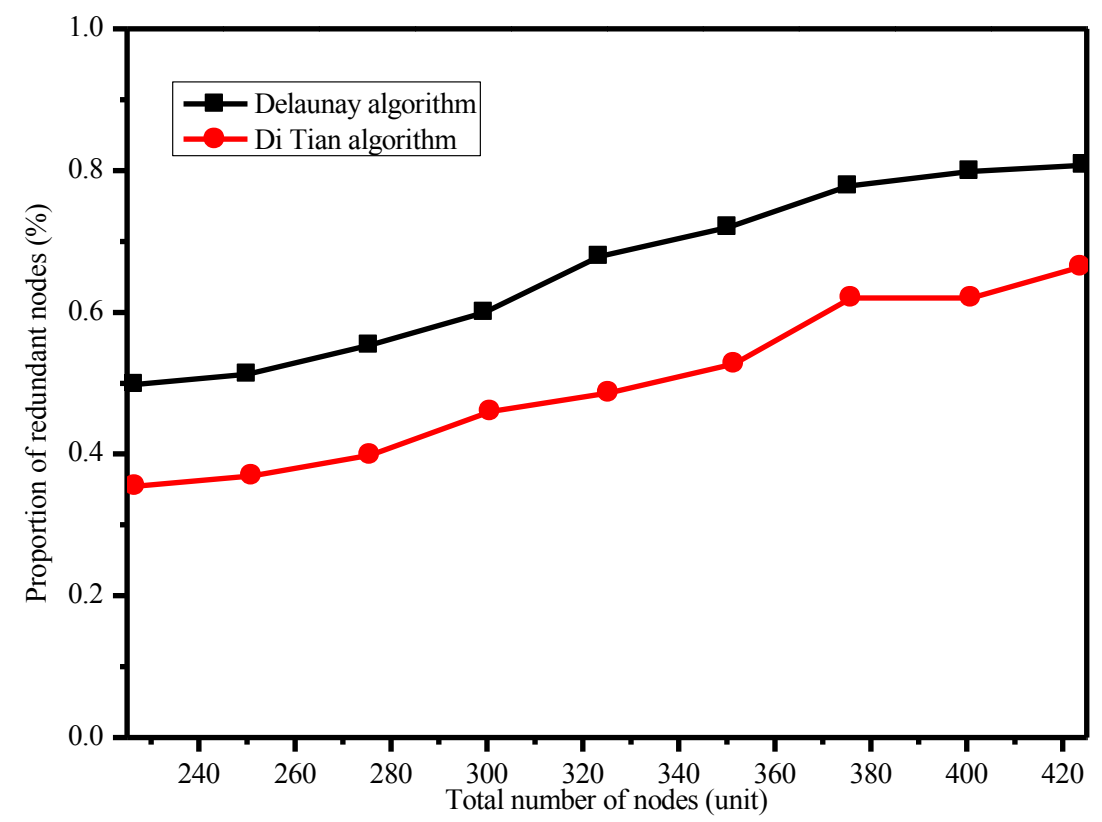

Fig. 8. Proportion of redundant nodes

It can be seen from Figure 7 that, the Di Tian algorithm and the algorithm results are with the increase in the number of nodes for WSNs, the proportion of redundant nodes is increasing. It is mainly because that the dispenser density of nodes increases, and the coverage holes under the initial conditions reduce, resulting in the increasing proportion of redundant nodes. For dispensing the same number of nodes, the number of redundant nodes judged by the algorithm in this paper is much more than that of Di Tian. 


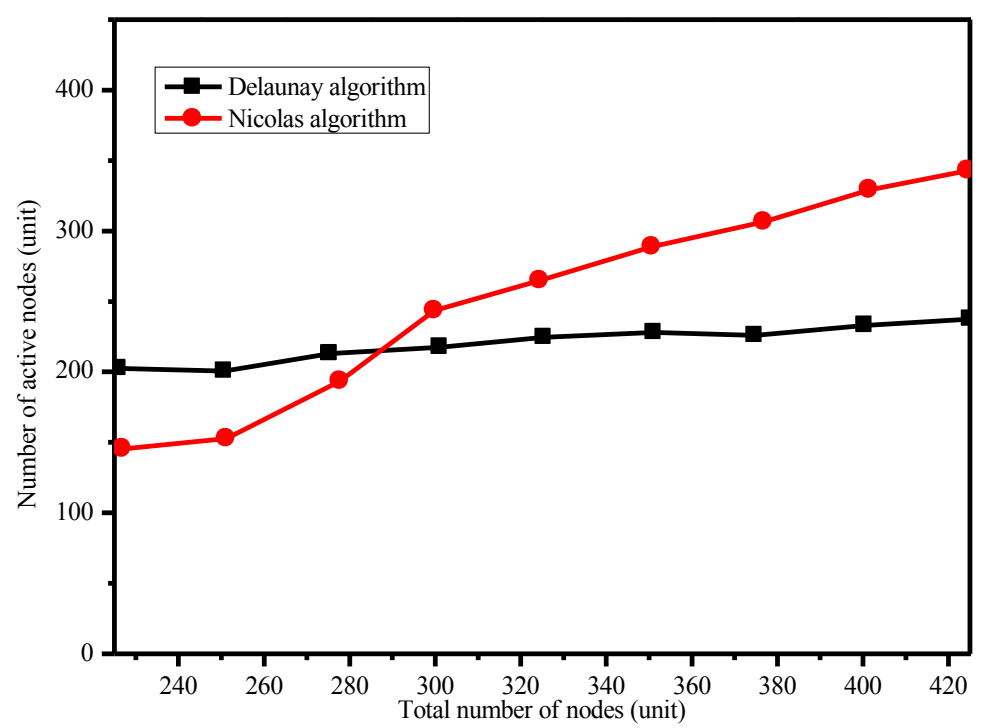

Fig. 9. Number of active nodes

It can be seen from Figure 8 that, for different number of redundant nodes in the same area, the number of active nodes obtained by the algorithm in this paper begins to increase, but the change is small, and tends to be stable. It is mainly because for the same coverage area, as long as there is a certain number of active nodes can it achieve full coverage. As a result, the number of active nodes chosen will not increase with the increase of dispenser nodes, and the number of active nodes obtained by Nicolas algorithm continuously increase with the increase of nodes, which means that there are still a large number of dormant nodes working in the network.

\section{Conclusion}

In this paper, for the node dormancy problems in the high density randomly dispersed sensor network, we put forward a new redundant node judgment and dormancy algorithm based on local Delaunay triangle graph. The algorithm is divided into two steps, namely, distinguishing the redundant nodes and selecting the dormant nodes. The premise of the algorithm is to construct the local Delaunay triangulation graph of the neighbor nodes, nodes according to the information of the graph to judge whether itself is off redundant nodes, and then to determine the independent redundant nodes and the redundant nodes and the dependence. The experimental results showed that the algorithm can effectively judge the redundant nodes in the network and make sure that some redundant nodes enter the dormant state in the case of ensuring the initial coverage situation unchanged. Compared with other algorithms, it is shown that the number of redundant nodes judged by the algorithm in this paper is more than that of the existing algorithm. In addition, in the case of dispersing different number of nodes in the same area, the number of active nodes obtained by the algorithm is very small 
in change, which can achieve coverage of monitoring area with fewer nodes. The algorithm is suitable for large networks with many nodes, and it has certain practical value.

\section{$7 \quad$ References}

[1] Aloi, G., Bedogni, L., Bononi, L., Briante, O., Felice, M. D., \& Loscrì, V., et al. (2015). Stem-net: how to deploy a self-organizing network of mobile end-user devices for emergency communication. Computer Communications, 60, 12-27. https://doi.org/10.1016/ j.comcom.2015.01.023

[2] Javadhosseini, M., Hosseinalipour, A., \& Sabamoniri, S. (2014). A new approach for covering wireless sensor networks with optimum number of nodes in order to prolonging network lifetime. International Journal of Computer Applications, 89(6), 37-41. https://doi.org/10.5120/15509-4282

[3] Lanteigne, E., \& Jnifene, A. (2014). Biologically inspired node generation algorithm for path planning of hyper-redundant manipulators using probabilistic roadmap. International Journal of Automation and Computing, 11(2), 153-161. https://doi.org/10.1007/s11633$\underline{014-0777-6}$

[4] Shao, C. K. (2013). Application of wireless sensor technology in the training of martial arts and specification motion detection. Advanced Materials Research, 791-793, 926-930. https://doi.org/10.4028/www.scientific.net/AMR.791-793.926

[5] Tian, J., Gao, M., \& Ge, G. (2016). Wireless sensor network node optimal coverage based on improved genetic algorithm and binary ant colony algorithm. EURASIP Journal on Wireless Communications and Networking, 2016(1), 1-11. https://doi.org/10.1186/s13638016-0605-5

[6] Wagner, D., Vogt, S., Jamal, F. I., Guha, S., Wenger, C., \& Wessel, J., et al. (2016). Application of microwave sensor technology in cardiovascular disease for plaque detection. Current Directions in Biomedical Engineering, 2(1), 273-277. https://doi.org/10.1515/cdb me-2016-0061

[7] Xu, D., \& Wang, K. (2015). An adaptive traffic mac protocol based on correlation of nodes. EURASIP Journal on Wireless Communications and Networking, 2015(1), 258. https://doi.org/10.1186/s13638-015-0488-x

[8] Zhao, X. (2014). Biochemical and molecular changes associated with heteroxylan biosynthesis in neolamarckia cadamba (rubiaceae) during xylogenesis. Frontiers in Plant Science, 5(602), 14.

\section{Authors}

Bo Feng is Associate Professor at Shaanxi University of Science \&Technology, Shaanxi Xi'an, China (ckmtvxo3434@126.com).

Wei Tang is Associate Professor at Shaanxi University of Science \&Technology, Shaanxi Xi’an, China (yicliuy8558646@126.com).

Guofa Guo is with Shaanxi University of Science \&Technology, Shaanxi Xi'an, China (xavrh752594@126.com).

Article submitted 21 April 2017. Published as resubmitted by the authors 23 May 2017. 